\title{
Acute respiratory failure and the kinetics of neutrophil recovery in pediatric hematopoietic cell transplantation: a multicenter study
}

\author{
J. R. Moffet ${ }^{1}$ - K. M. Mahadeo ${ }^{2}$ J. McArthur ${ }^{3}$ - D. D. Hsing ${ }^{4}$ - S. J. Gertz ${ }^{5}$ L. S. Smith ${ }^{6} \cdot$ A. Loomis ${ }^{7}$. J. C. Fitzgerald ${ }^{8}$. \\ M. E. Nitu' ${ }^{9}$ C. N. Duncan ${ }^{10}$ - M. W. Hall ${ }^{11}$ - E. L. Pinos ${ }^{12}$ - R. F. Tamburro ${ }^{12}$ - R. A. Simmons ${ }^{13} \cdot$ J. Troy $^{1}$. \\ I. M. Cheifetz ${ }^{14}$ - C. M. Rowan ${ }^{9} \cdot$ Investigators of the Pediatric Acute Lung Injury and Sepsis Network
}

Received: 10 February 2019 / Revised: 9 July 2019 / Accepted: 10 August 2019 / Published online: 16 September 2019

(c) The Author(s), under exclusive licence to Springer Nature Limited 2019

\begin{abstract}
In this multicenter study, we investigated the kinetics of neutrophil recovery in relation to acuity and survival among 125 children undergoing allogeneic hematopoietic cell transplantation (allo-HCT) who required invasive mechanical ventilation (IMV). Recovery of neutrophils, whether prior to or after initiation of IMV, was associated with a significantly decreased risk of death relative to never achieving neutrophil recovery. A transient increase in acuity (by oxygenation index and vasopressor requirements) occurred among a subset of the patients who achieved neutrophil recovery after initiation of IMV; $61.5 \%$ of these patients survived to discharge from the intensive care unit (ICU). Improved survival among patients who subsequently achieved neutrophil recovery on IMV was not limited to those with peri-engraftment respiratory distress syndrome. The presence of a respiratory pathogen did not affect the risk of death while on IMV but was associated with an increased length of IMV $(p<0.01)$. Among patients undergoing HCT who develop respiratory failure and require advanced therapeutic support, neutrophil recovery at time of IMV and/or presence of a respiratory pathogen should not be used as determining factors when counseling families about survival.
\end{abstract}

These authors contributed equally: J. R. Moffet, K. M. Mahadeo

J. R. Moffet

jerelyn.moffet@duke.edu

1 Department of Pediatrics, Division of Blood and Marrow Transplant, Duke Children's Hospital, Duke University, Durham, NC, USA

2 Department of Pediatrics, Stem Cell Transplantation and Cellular Therapy, University of Texas at MD Anderson Cancer Center, Houston, TX, USA

3 Department of Pediatrics, Division of Critical Care, St. Jude's Children's Research Hospital, Memphis, TN, USA

4 Department of Pediatrics, Division of Critical Care, Weil Cornell Medical College, New York Presbyterian Hospital, New York City, NY, USA

5 Department of Pediatrics, St. Barnabas Medical Center, Livingston, NJ, USA

6 Department of Pediatrics, Division of Pediatric Critical Care Medicine, Seattle Children's Hospital, University of Washington, Seattle, WA, USA

7 Department of Pediatrics, Division of Critical Care, Masonic Children's Hospital, University of Minnesota, Minneapolis, MN, USA
8 Department of Anesthesiology and Critical Care, Division of Critical Care, Children's Hospital of Philadelphia, University of Pennsylvania Perelman School of Medicine, Philadelphia, PA, USA

9 Department of Pediatrics, Division of Critical Care, Riley Hospital for Children, Indiana University School of Medicine, Indianapolis, IN, USA

10 Department of Pediatrics, Pediatric Oncology, Dana-Farber Cancer Institute Harvard University, Boston, MA, USA

11 Department of Pediatrics, Division of Critical Care, Nationwide Children's Hospital, The Ohio State University, Columbus, OH, USA

12 Department of Pediatrics, Division of Critical Care, Penn State Hershey Children's Hospital, Pennsylvania State University College of Medicine, Hershey, PA, USA

13 Duke CTSI Biostatistics, Epidemiology and Research Design (BERD) Methods Core, Department of Biostatistics \& Bioinformatics, Duke University, Durham, NC, USA

14 Department of Pediatrics, Division of Critical Care, Duke Children's Hospital, Duke University, Durham, NC, USA 


\section{Introduction}

Among children undergoing allogeneic hematopoietic cell transplantation (allo-HCT), pulmonary complications are the leading indication for critical care interventions [1-7]. Guidelines published by the Pediatric Acute Lung Injury Consensus Conference have defined pediatric acute respiratory distress syndrome (PARDS) with stratification into mild, moderate, and severe [8]. When applied to HCT patients, those who met severe PARDS criteria remained in the intensive care unit (ICU) longer, had longer courses of mechanical ventilation and were more likely to die compared with non-HCT patients [7]. Immunodeficiency has been recognized as a risk factor for the development of and mortality from PARDS and the kinetics of white blood cell recovery may impact morbidity and mortality following HCT [9-15]. Children and families undergoing HCT may benefit from ongoing discussions regarding prognosis and goals of care. We hypothesized that among children with acute respiratory failure requiring invasive mechanical ventilation (IMV) following HCT, those who had not recovered neutrophils at the time of IMV would have worse outcomes than those who had recovered neutrophils at the time of IMV. We further hypothesized that the presence of a respiratory pathogen would affect clinical outcome. The objective of this study was to identify whether neutrophil recovery should be considered an important variable to add to hypoxemia metrics in stratifying risk of mortality in future studies of invasively ventilated children.

\section{Materials and methods}

This is an a priori secondary analysis of a retrospective, multicenter cohort of pediatric allogeneic HCT recipients transferred to ICU within the first 60 days post transplant and who required IMV. Only patients that were in the first 60 days of transplant were included in this analysis, which was focused on neutrophil recovery and survival. Each center obtained institutional review board approval prior to study participation. Centers contributed up to 25 of their most recent consecutive allo-HCT recipients requiring IMV between 2009 and 2014. All indications for allo-HCT were included. Study patients were limited to those between 1 month and 21 years of age. Patients were excluded if they were intubated for indications other than critical illness (e.g., intubated solely for procedures or for postoperative care). Two children were excluded from data collection due to a length of PICU stay prior to IMV of more than 100 days.

Data abstracted included demographic information, transplant indication, graft source, and number of transplants, status of neutrophil recovery at onset of IMV, indication for IMV, time from transplant to IMV, mode of ventilation, respiratory parameters, use of renal replacement therapy (RRT), vasoactive support, presence and identification of respiratory pathogen, and survival to ICU discharge. Neutrophil recovery was defined as the first of 3 consecutive days of an absolute neutrophil count $>500$ cells per microliter. Oxygenation index (OI) and oxygen saturation index (OSI) were used as markers of severity of hypoxemic respiratory failure (a higher index suggestive of severity $). \quad\left\{\mathrm{OI}=\left(\mathrm{Fio}_{2} \times\right.\right.$ mean airway pressure $\left.\times 100\right) /$ $\mathrm{PaO}_{2}$ and $\mathrm{OSI}=\left(\mathrm{Fio}_{2} \times\right.$ mean airway pressure $\left.\times 100\right) /$ $\mathrm{Spo}_{2}$. The OSI was calculated only when the $\mathrm{Spo}_{2}$ was $<97 \%\}[7,16]$.

\section{Statistical methods}

Time to death from any cause while on IMV, or discharge from the PICU, was analyzed using the Kaplan-Meier method and log-rank test. The impact of host, graft, and disease characteristics on risk of death while on IMV was analyzed using stepwise Cox proportional hazards regression modeling. The final model contained only the neutrophil engraftment status; all other factors were eliminated during stepwise model building. Time to neutrophil recovery was analyzed using the Fine and Gray model. Follow-up time for this analysis started on the date of transplant and ended at either the date of death prior to engraftment (competing risk) or the first of 3 consecutive days of ANC $>500$ cells per microliter (neutrophil recovery). Patients who never achieved neutrophil recovery were classified as having graft failure (competing risk) at day 42 post transplant. Model assumptions were verified graphically. All analyses were conducted using SAS v9.4 (SAS Institute, Cary, NC).

\section{Results}

A total of 125 patients from 12 centers were included in this study. Patient demographics are summarized in Table 1. The median age was $7.3(0.2-20.9)$ years. Among this cohort, this was the first HCT for $82 \%$ of children, $47 \%$ received bone marrow as their stem cell source with the majority being from an unrelated donor $(79 \%)$. The median time from HCT to IMV was 23 (0-69) days. Those who had neutrophil recovery prior to the development of respiratory failure, had a longer time from HCT to IMV $(p<0.001)$. Respiratory distress was the primary reason for ICU admission $(86.4 \%)$ followed by hemodynamic instability. The majority of children $(76 \%)$ were intubated within $24 \mathrm{~h}$ of ICU admission.

As shown in Fig. 1, most children achieved neutrophil recovery prior to initiation of IMV $(n=72)$. Twenty-six 
Table 1 Patient and graft characteristics

\begin{tabular}{|c|c|c|c|c|c|}
\hline Patient characteristic & $\begin{array}{l}\text { Entire cohort } \\
(n=125)\end{array}$ & $\begin{array}{l}\text { Neutrophil } \\
\text { recovery at time } \\
\text { of IMV }(n=72)\end{array}$ & $\begin{array}{l}\text { Neutrophil } \\
\text { recovery after } \\
\text { initiation of IMV } \\
(n=26)\end{array}$ & $\begin{array}{l}\text { Never had } \\
\text { neutrophil } \\
\text { recovery } \\
(n=27)\end{array}$ & $p$ value \\
\hline $\begin{array}{l}\text { Age at ICU admission } \\
\text { (years) }\end{array}$ & $\begin{array}{l}7.3 \\
(0.2-20.9)\end{array}$ & $8.1(0.4-20.9)$ & $7.1(0.2-20.8)$ & $7.3(0.2-20.9)$ & 0.7053 \\
\hline First HCT & $102(81.6 \%)$ & $58(80.6 \%)$ & $24(92.3 \%)$ & $20(74.1 \%)$ & 0.2169 \\
\hline Transplant source & & & & & 0.0424 \\
\hline Bone marrow & $59(47.2 \%)$ & $33(45.8 \%)$ & $15(57.7 \%)$ & $11(40.7 \%)$ & \\
\hline Cord & $49(39.2 \%)$ & $24(33.3 \%)$ & $10(38.5 \%)$ & $15(55.6 \%)$ & \\
\hline Peripheral blood & $17(13.6 \%)$ & $15(20.8 \%)$ & $1(3.8 \%)$ & $1(3.7 \%)$ & \\
\hline Related donor & $26(20.8 \%)$ & $21(29.2 \%)$ & $1(3.8 \%)$ & $4(14.8 \%)$ & 0.0167 \\
\hline Malignant diagnosis & $68(54.4 \%)$ & $44(61.1 \%)$ & $15(57.7 \%)$ & $9(33.3 \%)$ & 0.0439 \\
\hline \multicolumn{6}{|c|}{ Diagnosis leading to transplant } \\
\hline $\begin{array}{l}\text { Acute lymphoblastic } \\
\text { leukemia }\end{array}$ & $31(25 \%)$ & $19(26 \%)$ & $8(31 \%)$ & $4(15 \%)$ & 0.3606 \\
\hline Acute myeloid leukemia & $25(20 \%)$ & $16(22 \%)$ & $6(23 \%)$ & $3(11 \%)$ & 0.4253 \\
\hline $\begin{array}{l}\text { Primary } \\
\text { immunodeficiency }\end{array}$ & $18(14 \%)$ & $11(15 \%)$ & $3(12 \%)$ & $4(15 \%)$ & 0.9413 \\
\hline $\begin{array}{l}\text { Hemophagocytic } \\
\text { lymphohistiocytosis }\end{array}$ & $13(10 \%)$ & $5(7 \%)$ & $3(12 \%)$ & $5(19 \%)$ & 0.2156 \\
\hline Bone marrow suppression & $12(10 \%)$ & $6(8 \%)$ & $1(4 \%)$ & $5(19 \%)$ & 0.2311 \\
\hline Metabolic/genetic & $10(8 \%)$ & $3(4 \%)$ & $3(12 \%)$ & $4(15 \%)$ & 0.1452 \\
\hline $\begin{array}{l}\text { Myelodysplastic } \\
\text { syndrome }\end{array}$ & $6(5 \%)$ & $6(8 \%)$ & $0(0 \%)$ & $0(0 \%)$ & 0.1330 \\
\hline Lymphomas & $5(4 \%)$ & $3(4 \%)$ & $1(4 \%)$ & $1(4 \%)$ & 0.9999 \\
\hline Hemoglobinopathies & $2(2 \%)$ & $2(3 \%)$ & $0(0 \%)$ & $0(0 \%)$ & 0.9999 \\
\hline Other/not specified & $3(2 \%)$ & $1(1 \%)$ & $1(4 \%)$ & $1(4 \%)$ & 0.3860 \\
\hline Days since $\mathrm{HCT}$ at IMV & $\begin{array}{l}23 \\
(0.0-69.0)\end{array}$ & $36(8-69)$ & $12(5-47)$ & $12(0-57)$ & $<0.0001$ \\
\hline \multicolumn{6}{|l|}{ Present at PICU admission } \\
\hline Respiratory distress & $108(86.4 \%)$ & $59(82.0 \%)$ & $25(96.2 \%)$ & $24(88.9 \%)$ & 0.1769 \\
\hline $\begin{array}{l}\text { Hemodynamic } \\
\text { instability }\end{array}$ & $19(15.2 \%)$ & $10(13.9 \%)$ & $2(7.7 \%)$ & $7(26.0 \%)$ & 0.1618 \\
\hline $\begin{array}{l}\text { Altered mental status or } \\
\text { seizures }\end{array}$ & $11(8.8 \%)$ & $8(11.1 \%)$ & $1(3.8 \%)$ & $2(7.4 \%)$ & 0.5119 \\
\hline $\begin{array}{l}\text { Respiratory pathogen } \\
\text { identified }\end{array}$ & $43(34.4 \%)$ & $25(34.7 \%)$ & $11(42.3 \%)$ & $7(25.9 \%)$ & 0.4532 \\
\hline $\begin{array}{l}\text { Receiving supplemental } \\
\mathrm{O}_{2} 7 \text { days prior to IMV }\end{array}$ & $46(36.8 \%)$ & $32(44.4 \%)$ & $8(30.8 \%)$ & $6(22.2 \%)$ & 0.0962 \\
\hline
\end{tabular}

Results are presented in medians with (ranges) or in counts with (percentage)

patients achieved neutrophil recovery while on IMV and 27 children never achieved neutrophil recovery. The following factors were associated with greater likelihood of neutrophil recovery at time of IMV ( $p<0.05$ for all): peripheral blood graft source, having a related donor, and malignant diagnosis as indication for HCT. Patients who were further from HCT at time of IMV were also more likely to have achieved neutrophil recovery $(p=<0.005)$. A total of 70 patients $(56 \%)$ died prior to ICU discharge.

\section{Neutrophil recovery and survival to ICU discharge}

ICU survivors were intubated earlier in their transplant course [median 10.5 days (2-24) vs. 15.5 (5-47) than nonsurvivors $(p=0.01)]$ and had a shorter ventilation course [median 16.5 days $(4-46)$ vs. 34.5 days (1-66) $p=$ $0.02]$. Age was not associated with ICU survival $(p=0.1)$. Results of our Kaplan-Meier analysis showed that survival was significantly different according to neutrophil recovery 


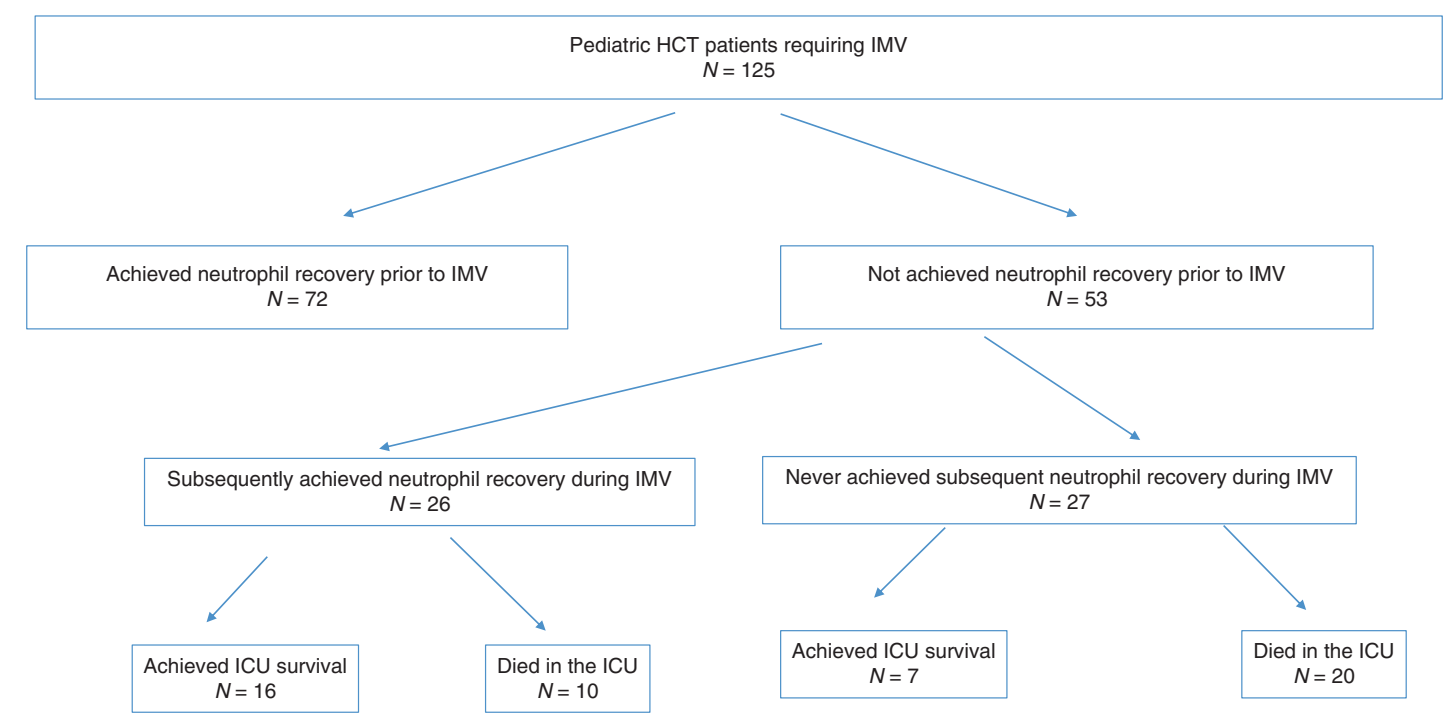

Fig. 1 Neutrophil recovery while on IMV

status $(p<0.0001)$. Patients who recovered neutrophils at any time during their ICU course had better survival than patients who never recovered neutrophils. Patients who had already achieved neutrophil recovery at the time of IMV fared worse than patients who recovered on the ventilator. Stepwise Cox proportional hazards regression modeling did not identify any additional host, graft, or disease-related predictors of death. Therefore, model results largely echoed results of the Kaplan-Meier analysis (Table 2). The greatest reduction in risk of death (relative to not recovering neutrophils) was observed in patients who achieved neutrophil recovery while on IMV (HR $=0.14,95 \%$ CI: $0.06,0.32$, $p<0.0001$ ) followed by patients who had recovered neutrophils at the time of IMV (HR $=0.33,95 \%$ CI: $0.18,0.59$, $p<0.0001$ ). Direct comparison of the two groups of patients who recovered neutrophils was also statistically significant $(P=0.02)$ and showed a higher risk of death in those who had achieved neutrophil recovery prior to institution of IMV compared with those who achieved neutrophil recovery after IMV (HR $=2.27,95 \%$ CI: 1.13, 4.57).

\section{The impact of neutrophil recovery on severity of illness}

OI was used a marker of severity of oxygenation failure and the use of vasoactive agents was used as a means to assess hemodynamic instability. Neutrophil recovery while receiving IMV was associated with transiently worsened acuity of illness by these measures. The median OI 12-24 h prior to neutrophil recovery was 9.5 . This value increased to 15.6 at time of recovery demonstrating a worsening in oxygenation failure. In addition, the percent of patients requiring vasoactive support increased from $20 \%$ at $12-24 \mathrm{~h}$ prior to neutrophil recovery to $36 \%$ at time of recovery.
Neither of these changes was sustained, with both values trending back toward preneutrophil recovery levels by 24-48 h post recovery. More than half $(56 \%)$ of children who achieved neutrophil recovery while in the ICU required RRT compared with $33 \%$ of children who achieved recovery prior to ICU admission and $19 \%$ of children who never achieved recovery $(p=0.02)$. There was no difference in survival in children who received or did not receive $\operatorname{RRT}(p=0.10)$.

\section{Predictors of neutrophil recovery in the ICU}

Graft source, indication for transplant, the presence of a respiratory pathogen, and demographic measures analyzed were not associated with neutrophil recovery. A total of 16 of the 26 patients $(61.5 \%)$ who recovered neutrophils while receiving IMV survived to ICU discharge. Survivors among this group were younger at a median age of 1.94 (range: 0.17-19.78) years compared with nonsurvivors with a median age of 14.67 (range $0.33-20.78$ ) years.

\section{Respiratory pathogens and causes of respiratory failure}

The causes of respiratory failure among this cohort are various and are listed in Table 3. Among the entire cohort, 43 children $(34 \%)$ had at least one respiratory pathogen identified at some time-point in their ICU course. Children who received related donor transplants were significantly less likely to have tested positive for a respiratory pathogen $(p=0.04)$. Among the 43 children who tested positive for a respiratory pathogen (virus, bacteria, and/or fungus obtained from a tracheal aspirate, protected brush specimen, or bronchial alveolar lavage), the type of infection did not 
Table 2 Risk of death in the PICU: results of Cox proportional hazards regression modeling $(N=125)$

\begin{tabular}{lcc}
\hline & HR (95\% CI) & $P$ value \\
\hline $\begin{array}{l}\text { Achieved neutrophil recovery prior to institution of IMV vs. never achieved } \\
\text { neutrophil recovery }\end{array}$ & $0.33(0.18,0.59)$ & $<0.0001$ \\
$\begin{array}{l}\text { Achieved neutrophil recovery after institution of IMV vs. never achieved } \\
\text { neutrophil recovery }\end{array}$ & $0.14(0.06,0.32)$ & $<0.0001$ \\
$\begin{array}{l}\text { Achieved neutrophil recovery prior to institution of IMV vs. achieved } \\
\text { neutrophil recovery after IMV }\end{array}$ & $2.27(1.13,4.57)$ & 0.0214 \\
\hline
\end{tabular}

$H R$ hazard ratio, $C I$ confidence interval

Table 3 Causes of respiratory failure

\begin{tabular}{llllr}
\hline Cause of respiratory failure & $\begin{array}{l}\text { Entire cohort } \\
n=125\end{array}$ & $\begin{array}{l}\text { Neutrophil recovery prior } \\
\text { to IMV } n=72\end{array}$ & $\begin{array}{l}\text { Neutrophil recovery on } \\
\text { IMV } n=26\end{array}$ & $\begin{array}{l}\text { Never achieved neutrophil } \\
\text { recovery } n=27\end{array}$ \\
\hline Pneumonia/Pulmonary infection $^{\mathrm{a}}$ & $33(26 \%)$ & $19(26 \%)$ & $9(35 \%)$ & $5(19 \%)$ \\
Shock/Hemodynamic instability & $26(21 \%)$ & $11(15 \%)$ & $3(12 \%)$ & $12(44 \%)$ \\
Pulmonary hemorrhage & $22(18 \%)$ & $12(17 \%)$ & $6(23 \%)$ & $4(15 \%)$ \\
Fluid overload & $19(15 \%)$ & $12(17 \%)$ & $4(15 \%)$ & $3(11 \%)$ \\
Hypoxia without identified infection & $6(5 \%)$ & $3(4 \%)$ & $1(4 \%)$ & $0(0 \%)$ \\
or other cause & & $4(6 \%)$ & $0(0 \%)$ & $0(0 \%)$ \\
Altered mental status & $4(3 \%)$ & $2(3 \%)$ & $0(0 \%)$ & $0(0 \%)$ \\
Idiopathic pneumonia syndrome & $2(2 \%)$ & $0(0 \%)$ & $2(8 \%)$ & $0(0 \%)$ \\
Upper airway obstruction/mucositis & $2(2 \%)$ & $1(1 \%)$ & $0(0 \%)$ & $0(0 \%)$ \\
Pericardial effusion & $1(1 \%)$ & $1(1 \%)$ & $1(4 \%)$ & $1(4 \%)$ \\
Pulmonary hypertension & $1(1 \%)$ & $7(10 \%)$ &
\end{tabular}

Data are presented in frequencies and $(\%)$ with percentages rounded to the nearest whole number

${ }^{\text {a }}$ Pulmonary infections included infections from any pathogen (ie bacterial, viral or fungal pathogens)

${ }^{\mathrm{b}}$ These subjects had hypoxia or ARDS as the cause of respiratory failure with other clear etiology listed on this table

impact survival $(p=0.38)$ and there was no difference in survival between children who achieved neutrophil engraftment (36\%) and those who had not (33\%) $(p=0.28)$. In a Cox proportional hazards model, the presence of any respiratory pathogen did not affect the risk of death while on IMV (HR: 0.63, 95\% CI: 0.37, 1.05; $p$-value $=0.07$ ). However, the presence of an identified respiratory pathogen was associated with an increased length of IMV (median: 17 days, interquartile range $[\mathrm{IQR}]=21$ days) compared with those who had nonidentified respiratory pathogen (median: 8.5 days, $\mathrm{IQR}=15$ days) $(p=0.0003)$.

\section{Discussion}

Mortality among pediatric HCT patients who require critical care support remains unacceptably high; the absence of neutrophils may add a layer of prognostic difficulty to an already complicated clinical situation [3-6]. Sorror et al. developed a hematopoietic cell transplantation-specific comorbidity index (HCT-CI) which aids as a pre-HCT predictor of nonrelapse mortality and overall survival among adult and pediatric HCT patients [17, 18]. While the HCT-CI is helpful in the pre-HCT setting to help quantify the risk of mortality from transplantation-related complications, there is a paucity of data to guide prognostication among pediatric intensivists and HCT physicians for children undergoing HCT who require critical care interventions.

In this study, we sought to identify whether neutrophil recovery impacts survival of children receiving IMV following HCT and identify factors that could result in improved outcomes. Our findings suggest that critically ill pediatric HCT patients who require IMV can be supported through neutrophil recovery. Indeed, $61.5 \%$ of children without neutrophil recovery at the time of intubation who subsequently achieved recovery also achieved ICU survival. This finding offers important management insight regarding children who require IMV following allo-HCT. While recovery of neutrophils did seem to worsen acuity as assessed by oxygenation failure, need for vasoactive agents and need for RRT, most of these acuity measures were temporary, returning to baseline within 1-2 days. In fact, more patients who achieved neutrophil recovery following 
IMV required RRT compared with those who achieved recovery prior. Perhaps this is secondary to the cytokine release, inflammatory state associated with neutrophil recovery leading to generalized capillary leak that improved with RRT (with fluid management or cytokine removal by RRT) [19]. Regardless, this data would further support the use of aggressive critical care interventions during this time as the increased acuity is likely short lived and could result in survival. The most common cause of respiratory failure among patients who achieved neutrophil recovery on IMV was pulmonary infection; among patients who never achieved recovery, shock/cardiovascular collapse was the most common indication. These findings suggest that improved survival among patients who subsequently achieved neutrophil recovery on IMV was not limited to those patients with peri-engraftment respiratory distress syndrome.

It is interesting that the children who were admitted to the ICU with neutrophil recovery had poorer survival than those who achieved neutrophil recovery while intubated. Our dataset is limited by the lack of detailed information of the transplant course and previously treated infections prior to ICU admission. However, while it is challenging to determine conclusively why this is the case, one may speculate that diseases contributing to respiratory failure may be a contributing factor. Noninfectious causes of respiratory failure such as bronchiolitis obliterans and cryptogenic organizing pneumonia tend to occur later in the transplant course, after engraftment. These noninfectious causes are challenging to diagnose, demonstrated by the fact that not one of the subjects in this study had a specific noninfectious diagnosis. Not only does the diagnosis pose a problem, but the treatment for many noninfectious pulmonary complications are nonspecific leading to poor outcome. Perhaps, children who present critically ill after neutrophil recovery, are at higher risk for these types of respiratory complications, which contributes to higher mortality. Our current study contributes to the understanding of respiratory failure among children undergoing HCT and should help guide the application of advanced technological therapies for this population. The critical care of the mechanically ventilated pediatric HCT patient is highly variable [18]. There are often strong and divergent perspectives from both specialties providing care. The high mortality rate makes the decision to institute aggressive mechanical support such as intubation, RRT, and extracorporeal membrane oxygenation difficult. Our cohort would support that the lack of neutrophils at the time respiratory failure develops is not associated with increased mortality. These patients may benefit from early and aggressive critical care interventions (including RRT) particularly when there are signs of impending neutrophil recovery.
Improved outcomes associated with neutrophil recovery raise the question of the utility of granulocyte infusions while on mechanical ventilation, particularly in the setting of an active infection. In this cohort, presence of a respiratory pathogen was not associated with increased mortality while on IMV. It is possible that neutrophil recovery status was a mitigating factor against presence of a respiratory pathogen. Some have suggested that the infusion of donor or third-party granulocytes may be useful early in the prophylaxis or treatment of severe neutropenic sepsis among patients undergoing allo-HCT [20-25].

Those that never achieved neutrophil recovery had dismal survival. At some point during the course of mechanical ventilation, failure to recover neutrophils, while not the sole prognostic sign for survival, should serve as one indication to discuss goals of care. The lack of neutrophil recovery may become even more important when it is combined with additional variables that have been prognostic for respiratory failure mortality such as OI, peak inspiratory pressure, and length of mechanical ventilation. The challenge remains timing of this conversation. This study begins the investigation to determine critical care factors associated with neutrophil recovery. Factors that suggest higher acuity, such as the need for CPR and use of high frequency oscillatory ventilation, were associated with a decreased chance of neutrophil recovery. Perhaps these factors suggest that the patient is too sick to be supported until neutrophil recovery can be achieved. However, it could also be that severe critical illness itself is delaying neutrophil recovery. The etiology is unclear but these factors should be considered in future prospective studies.

Our study is limited by its retrospective design. For example, while we were able to assess the impact of neutrophil recovery on clinical outcomes, we were unable to determine the impact of donor engraftment, as donor chimerism was not collected as part of the original dataset. We also did not collect information regarding veno-occlusive disease, granulocyte transfusions, growth factor support, and the impact of these on outcomes. While the multicenter nature of this large pediatric cohort allows generalizability of results, our findings would best be validated in a prospective setting.

Taken together, among patients undergoing HCT who develop respiratory failure and require advanced therapeutic support, the status of neutrophil recovery may significantly impact survival. Children who are expected to achieve neutrophil recovery following IMV may show worsening acuity that is temporary and should be considered for aggressive support measures. In addition, management teams should anticipate increased need for support in the peri-engraftment period. Among Pediatric HCT patients with the first signs of respiratory distress, a trial of noninvasive ventilation (with positive pressure) is reasonable, 
although intubation should not be delayed when indicated $[6,7]$. Among patients with early signs of respiratory distress and suspicion of an infection, early bronchoscopy may allow for prompt and pathogen specific treatment [26]. This is particularly important, as a respiratory pathogen in this cohort did not increase mortality, it was associated with a longer length of ventilation.

Our study identifies opportunities to improve survival among patients with historically poorer outcomes. Among patients undergoing HCT who develop respiratory failure and require advanced therapeutic support, neutrophil recovery at time of IMV and/or presence of a respiratory pathogen should not be used as determining factors when counseling families about survival. It is important that families be provided with comprehensive information that includes the limitations of our experience to arrive at informed consent.

Acknowledgements The authors would like to recognize the investigators of the Pediatric Acute Lung Injury and Sepsis Network for their support in this research.

Authors contributions CR designed the study. JM, KMM, CR, and CD analyzed data. JT and RS performed statistical analysis. JM, $\mathrm{KMM}, \mathrm{CD}, \mathrm{IC}$, and CR wrote the manuscript. SG, MH, AL, MN, JF, and JMc collected data and reviewed the manuscript. All authors made significant contributions to the manuscript.

\section{Compliance with ethical standards}

Conflict of interest The authors declare that they have no conflict of interest.

Publisher's note Springer Nature remains neutral with regard to jurisdictional claims in published maps and institutional affiliations.

\section{References}

1. Balit CR, Horan R, Dorofaeff T, Frndova H, Doyle J, Cox PN, et al. Pediatric hematopoietic stem cell transplant and intensive care: have things changed? Pediatr Crit Care Med. 2016; 3:109-116. 10.1097

2. Duncan CN, Lehmann LE, Cheifetz IM, Greathouse K, Haight A, Hall M, et al. Clinical outcomes of children receiving intensive cardiopulmonary support during hematopoietic stem cell transplant. Pediatr Crit Care Med. 2013;3:261-267. 10.1097

3. Chima RS, Daniels RC, Kim MO, Li D, Wheeler DS, Davies SM, et al. Improved outcomes for stem cell transplant recipients requiring pediatric intensive care. Pediatr Crit Care Med. 2012; 13:336-342.

4. Diaz MA, Vicent MG, Prudencio M, Rodriguez R, Marin C, Serrano A, et al. Predicting factors for admission to an intensive care unit and clinical outcome in pediatric patients receiving hematopoietic stem cell transplantation. Haematologica. 2002; 87:292-298.

5. Lamas A, Otheo E, Ros P, Vazquez JL, Maldonado MS, Munoz A, et al. Prognosis of child recipients of hematopoietic stem cell transplantation requiring intensive care. Intensive Care Med. 2003;29:91-96.

6. Rowan CM, Gertz SJ, McArthur J, Fitzgerald JC, Nitu ME, Loomis A, et al. Invasive mechanical ventilation and mortality in pediatric hematopoietic stem cell transplantation: a multicenter study. Pediatr Crit Care Med. 2016;4:294-302. https://doi.org/10. 1097/PCC0000000000000673

7. Rowan CM, Smith LS, Loomis A, McArthur J, Gertz SJ, Fitzgerald JC, et al. Pediatric acute respiratory distress syndrome in pediatric allogeneic hematopoietic stem cell transplants-a multicenter study. Pediatr Crit Care Med. 2017;18:304-309.

8. Pediatric Acute Lung Injury Consensus Conference Group. Pediatric acute respiratory distress syndrome: consensus recommendations from the pediatric acute lung injury consensus conference. Pediatr Crit Care Med. 2015;16:428-439.

9. Flori H, Dahmer MK, Sapru A, Quasney MW. Comorbidities and assessment of severity of pediatric acute respiratory distress syndrome: proceedings from the pediatric acute lung injury consensus conference. Pediatr Crit Care Med. 2015;16:S41-50.

10. Erickson S, Schibler A, Numa A, Nuthall G, Yung M, Pascoe E, et al. Acute lung injury in pediatric intensive care in Australia and New Zealand: a prospective, multicenter, observational study. Pediatr Crit Care Med. 2007;8:317-323.

11. Zimmerman JJ, Akhtar SR, Caldwell E, Rubenfeld GD. Incidence and outcomes of pediatric acute lung injury. Pediatrics. 2009; 124:87-95.

12. Nichols DG, Walker LK, Wingard JR, Bender KS, Bezman M, Zahurak ML, et al. Predictors of acute respiratory failure after bone marrow transplantation in children. Crit Care Med. 1994; 22:1485-1491.

13. Santschi M, Jouvet P, Leclerc F, Gauvin F, Newth CJ, Carroll CL, et al. Acute lung injury in children: Therapeutic practice and feasibility of international clinical trials. Pediatr Crit Care Med. 2010;11:681-689.

14. Timmons OD, Havens PL, Fackler JC. Pediatric critical care study group, extracorporeal life support organization. Predicting death in pediatric patients with acute respiratory failure. Chest. 1995;108:789-797.

15. Kim HT, Frederick D, Armand P, Andler E, Kao G, Cutler C, et al. White blood cell recovery after allogeneic hematopoietic cell transplantation predicts clinical outcome. Am J Hematol. 2014; 89:591-597. 10.1002

16. Thomas NJ, Shaffer ML, Willson DF, Shih MC, Curley MA. Defining acute lung disease in children with the oxygenation saturation index. Pediatr Crit Care Med. 2010;11:12-17.

17. Sorror M, Maris M, Storb R, Baron F, Sandmaier BM, Maloney DG, et al. Hematopoietic cell transplantation (HCT)-specific comorbidity index: a new tool for risk assessment before allogeneic HCT. Blood. 2005;106:2912-2919.

18. Smith AR, Majhail NS, MacMillan ML, DeFor TE, Jodele S, Lehmann LE, et al. Hematopoietic cell transplantation comorbidity index predicts transplantation outcomes in pediatric patients. Blood. 2011;117:2728-34. 10.1182/blood-2010-08-303263

19. DiCarlo JV, Alexander SR, Argawal R, Shiffman JD. Continuous veno-venous hemofiltration may improve survival from acute respiratory distress syndrome after bone marrow transplantation or chemotherapy. J Pediatr Hematol Oncol. 2003;25:801-805.

20. Rowan CM, Nitu ME, Rigby MR. Inconsistencies in care of the pediatric hematopoietic stem cell transplant recipient with respiratory failure: opportunity for standardization and improved outcome. Pediatr Transpl. 2014;18:230-235.

21. Grigg A, Vecchi L, Bardy P, Szer J. G-CSF stimulated donor granulocyte collections for prophylaxis and therapy of neutropenic sepsis. Aust N Z J Med. 1996;26:813-818. 
22. Tsukada Y, Nagayama H, Mori T, Shimizu T, Sata N, Takayama $\mathrm{N}$, et al. Granulocyte transfusion as a treatment for enterococcal meningoencephalitis after allogeneic bone marrow transplantation from an unrelated donor. Bone Marrow Transpl. 2003;31:69-72. 10.1038

23. Robinson SP, Marks DI. Granulocyte transfusions in the G-CSF era. Where do we stand? Bone Marrow Transpl. 2004;34:839-846. 10.1038

24. Cugno C, Deola S, Filippini P, Stroncek DF, Rutella S. Granulocyte transfusions in children and adults with hematological malignancies: benefits and controversies. J Transl Med. 2015; 13:362. 10.1186

25. Valentini CG, Farina F, Pagano L, Teofili L. Granuloycte transfusions: a critical reappraisal. Biol Blood Marrow Transpl. 2017;23:2034-2041.

26. Shannon VR, Andersson BS, Lei X, Champlin RE, Kontoyiannis DP. Utility of early versus late fiberoptic bronchoscopy in the evaluation of new pulmonary infiltrates following hematopoietic stem cell transplantation. Bone Marrow Transpl. 2010;45:647-55. 